\title{
April 2018 Imaging Case of the Month
}

\author{
Robert W. Viggiano, MD* \\ Michael B. Gotway, MD**
*Pulmonary Department and **Department of Radiology
Mayo Clinic Arizona
Scottsdale, AZ USA

Clinical History: A 65-year-old non-smoking man with a past medical history significant for hyperlipidemia, hypertension, coronary artery disease, and pacemaker placement, presented for a routine medical evaluation.

The patient was allergic to penicillin, and his list of medications included aspirin, a diuretic, an ACE inhibitor, and a statin, in addition to over-the-counter vitamin supplements. Laboratory evaluation showed a normal complete blood count, electrolyte panel, and liver function tests. Frontal and chest radiography (Figure 1) was performed.

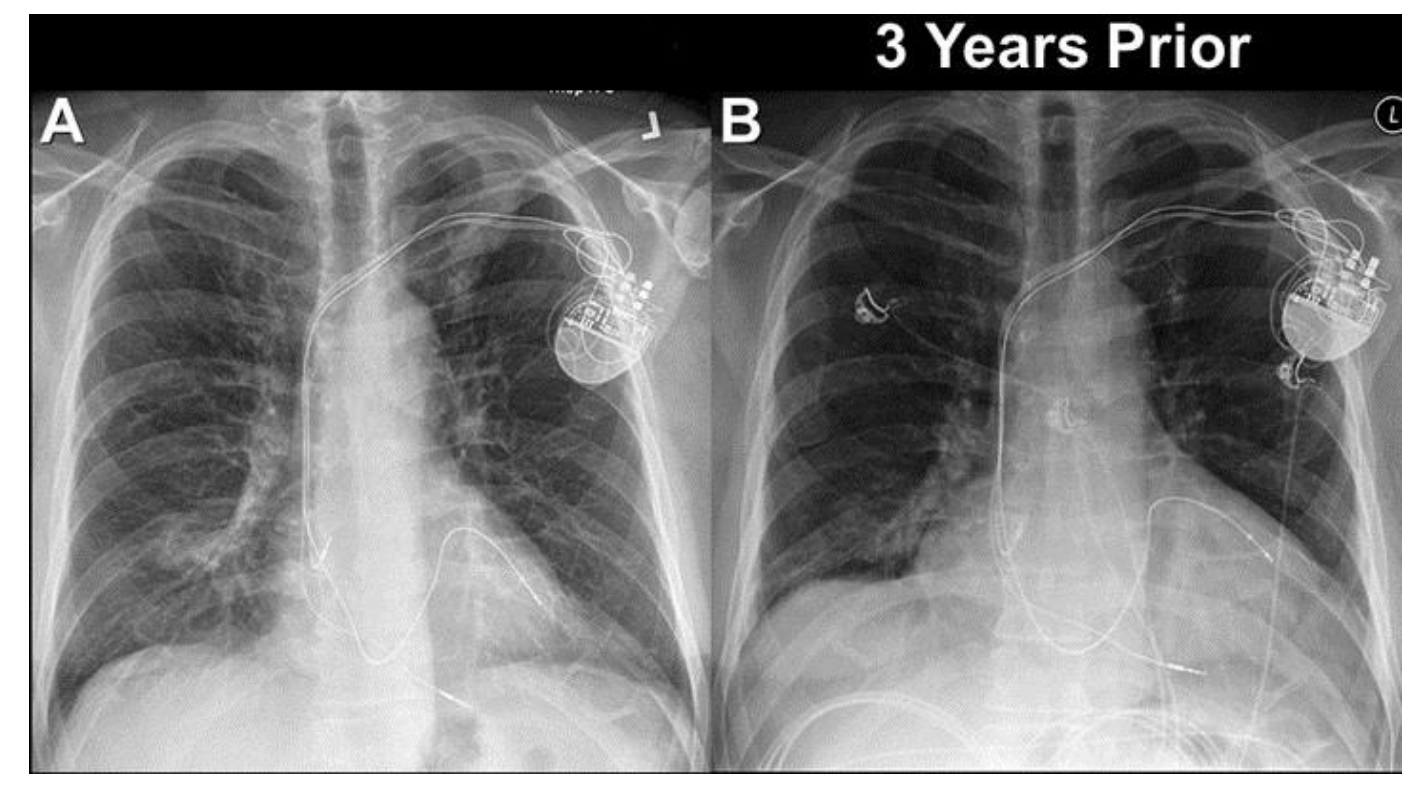

Figure 1. Frontal chest radiography performed at presentation $(A)$ and three years earlier (B).

Which of the following represents the most accurate assessment of the frontal chest imaging findings?

1. Chest frontal imaging shows a mediastinal mass

2. Chest frontal imaging shows bilateral peribronchial and mediastinal lymph node enlargement

3. Chest frontal imaging shows bilateral pleural fluid collections

4. Chest frontal imaging shows focal masses

5. Chest frontal imaging shows reduced lung volumes with basilar fibrotic changes 


\section{Correct! \\ 4. Chest frontal imaging shows focal masses}

The frontal radiograph shows bilateral pulmonary masses- one vague opacity in the left apex, the other projected over the right base. Both findings were not present on a chest radiograph performed 3 years earlier. Lung volumes appear normal and no findings suggesting interstitial lung disease are seen. The mediastinal and hilar / peribronchial contours are within no limits- no mediastinal mass or evidence of lymph node enlargement is present. No abnormality affecting the pleura is seen.

The patient underwent testing for exposure to fungal infection, including coccioidomycosis and histoplasmosis, both of which were unrevealing.

Which of the following represents the most appropriate management step for the evaluation of this patient?

1. Obtain ${ }^{18}$ FDG-PET scan

2. Obtain surgical lung biopsy

3. Obtain thoracic CT

4. Perform bronchoscopy

5. Treat presumptively with antibiotics and re-image in 4 weeks 


\section{Correct! \\ 3. Obtain thoracic CT}

Treating with antibiotics and performing follow up imaging is a reasonable approach when a bacterial infection is suspected, and, occasionally, bacterial infections can appear somewhat rounded- so-called "round pneumonia." This process tends to be encountered more commonly in children. The appearance of the opacities at chest radiography in an adult asymptomatic patient would be highly unusual for a bacterial infection. Performing ${ }^{18}$ FDG-PET scan is not unreasonable, but typically ${ }^{18}$ FDG-PET scans are most informative following morphologic characterization of pulmonary, pleural, or mediastinal abnormalities with thoracic CT. Surgical lung biopsy is inappropriately aggressive at this point, and bronchoscopy is premature, as the opacities at chest radiography should first be confirmed and characterized more thoroughly before invasive procedures are considered.

The patient underwent thoracic CT (Figure 2) for further characterization of the opacities detected at chest radiography.

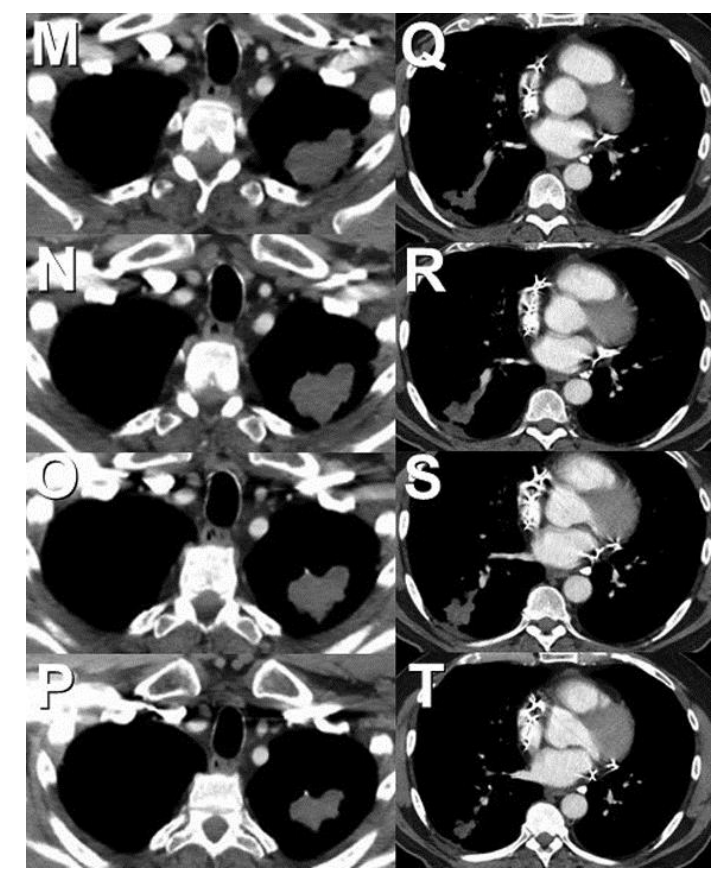

Figure 2. Axial enhanced thoracic CT displayed in soft tissue windows.

Which of the following represents the most accurate assessment of the thoracic CT findings?

1. Thoracic CT shows multifocal ground glass opacity

2. Thoracic CT shows cavitary pulmonary lesions

3. Thoracic CT shows two non-specific pulmonary masses

4. Thoracic CT shows numerous, small, miliary nodules

5. Thoracic CT shows fibrotic lung disease 


\section{Correct!}

\section{Thoracic CT shows two non-specific pulmonary masses}

The unenhanced thoracic CT confirms the two pulmonary masses detected at chest radiography, and also shows calcified mediastinal lymph nodes, but there is no evidence of cavitation, miliary nodules, or fibrotic lung disease.

Given the unenhanced thoracic CT findings, which of the following represents the most appropriate management step for the evaluation of this patient?

1. Obtain ${ }^{18}$ FDG-PET scan

2. Obtain surgical lung biopsy

3. Obtain thoracic MRI

4. Perform bronchoscopy

5. Repeat contrast-enhanced thoracic CT using a solitary pulmonary nodule protocol 


\section{Correct! \\ 1. Obtain 18FDG-PET scan}

Among the choices listed, ${ }^{18} \mathrm{FDG}-\mathrm{PET}$ scanning may be the best choice since two masses are present, and ${ }^{18} \mathrm{FDG}-\mathrm{PET}$ may provide data regarding which lesion should preferentially be targeted for intervention or may even disclose additional sites of disease that are more accessible to sampling. Repeating the CT using a specific nodule enhancement protocol would likely not yield management-altering results, although it is possible that one lesion would show enhancement whereas the other may not, directing intervention towards to enhancing nodule. However, because this protocol requires sequential scanning at a single chosen level within the center of the nodule, 4 times at 1-minute intervals, using this protocol to evaluate two separate masses widely spatially separated from one another, is not practical. Surgical lung biopsy remains premature as it is difficult to know which mass should be intervened upon. While bronchoscopy could be entertained, and it is possible to evaluate both masses at the same procedure, further non-invasive characterization would be capable of directing to bronchoscopic procedure more effectively. Thoracic MRI would not provide any information regarding the masses to what is already known with CT.

The patient underwent ${ }^{18}$ FDG-PET scanning (Figure 3).

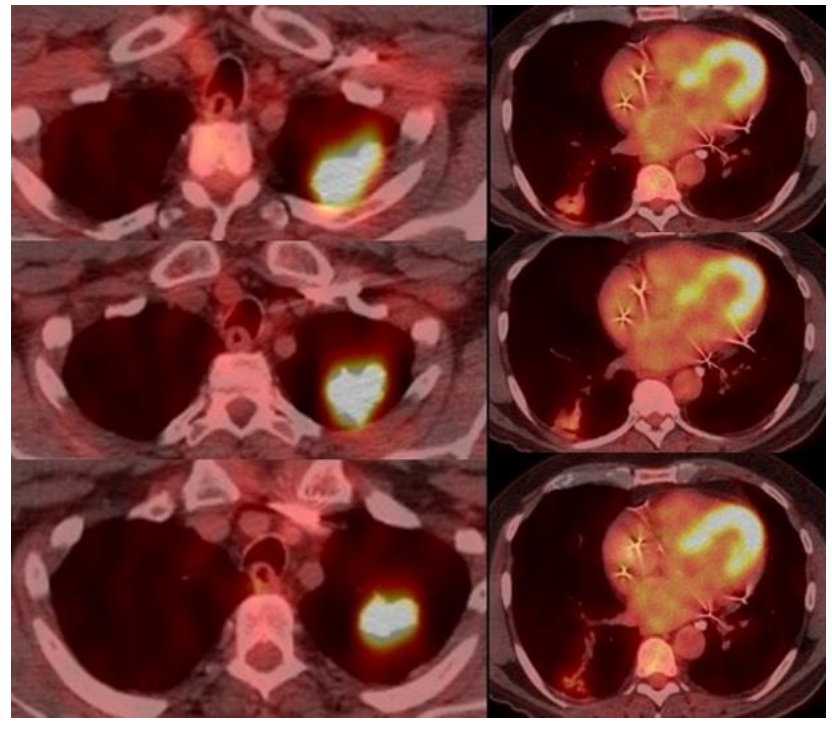

Figure 3. 18FDG-PET scan.

Which of the following represents the most accurate assessment of the ${ }^{18} \mathrm{FDG}$-PET findings?

1. ${ }^{18} \mathrm{FDG}-\mathrm{PET}$ is non-diagnostic owing to artifacts

2. ${ }^{18}$ FDG-PET scan shows both masses are intensely hypermetabolic

3. ${ }^{18} \mathrm{FDG}-\mathrm{PET}$ scan shows discrepant metabolism between the two masses

4. ${ }^{18} \mathrm{FDG}-\mathrm{PET}$ scan shows neither masse shows significant hypermetabolism

5. ${ }^{18} \mathrm{FDG}-\mathrm{PET}$ shows metabolically active mediastinal nodal uptake 


\section{Correct!}

\section{3. ${ }^{18}$ FDG-PET scan shows discrepant metabolism between the two masses}

The ${ }^{18} \mathrm{FDG}-\mathrm{PET}$ scan reveals discrepant metabolism within the two pulmonary masses, showing intense metabolic activity within the left apical mass, but only minimal metabolic activity within the right lower lobe mass. No abnormal tracer activity is seen within peribronchial or mediastinal lymph nodes, and no other sites of significant tracer utilization are present elsewhere inside or outside the thorax.

Given the data available, which of the following represents the most appropriate management step for the evaluation of this patient?

1. Obtain thoracoscopic surgical lung biopsy of the left apical mass

2. Obtain transthoracic percutaneous needle biopsy of the left apical mass

3. Perform ${ }^{68} \mathrm{Ga}$ - PET - CT dotatate scan

4. Perform bronchoscopy directed at the left apical mass

5. Serial imaging evaluation of the masses to exclude growth 


\section{Correct!}

\section{Obtain transthoracic percutaneous needle biopsy of the left apical mass}

${ }^{68} \mathrm{Ga}-\mathrm{PET}-\mathrm{CT}$ dotatate scanning is used for staging of neuroendocrine malignancies and would not play a role for this patient unless neuroendocrine malignancy is subsequently demonstrated. Surgical lung biopsy certainly could obtain tissue to establish the etiology of the left apical mass, but is unnecessarily invasive when other, less invasive, procedures may provide diagnostic material. Bronchoscopy is one such procedure but given the extreme apical location of the left upper lobe metabolically active mass, percutaneous transthoracic needle biopsy of the left apical mass would be a better choice for addressing this lesion. Note that the metabolic activity detected at ${ }^{18} \mathrm{FDG}-\mathrm{PET}$ scan suggests that diagnostic procedures should be directed preferentially at the left apical mass, as opposed to the right lower lobe mass. Serial CT evaluation for growth is not an appropriate management strategy for pulmonary lesions of this size.

The patient underwent navigational bronchoscopy of directed at the left apical mass, but only non-specific material was obtained. Subsequently, the patient underwent percutaneous transthoracic needle biopsy of the left apical lesion. The material obtained at biopsy showed fibrinous injury with necrosis and giant cells, with inflammation that was "vaguely granulomatous." No acid-fast or fungal organisms were identified. There was no evidence of malignancy. Culture of the biopsy material subsequently grew Mycobacterium avium-complex (MAC).

Given the data available, which of the following represents the most appropriate management step for the evaluation of this patient?

1. Obtain thoracoscopic surgical lung biopsy of the right lower lobe mass

2. Obtain transthoracic percutaneous needle biopsy of the right lower lobe mass

3. Perform bronchoscopy directed at the right lower lobe mass

4. Perform dynamic contrast-enhanced MRI of the right lower lobe mass

5. Treat for mycobacterial infection and observe 


\section{Correct! \\ 2. Obtain transthoracic percutaneous needle biopsy of the right lower lobe mass}

As noted previously, surgical biopsy of the lung masses is needlessly invasive when less aggressive options stand a good chance at establishing a diagnosis. Dynamic contrast-enhanced MRI is unlikely to add management-altering information. The right lower lobe mass shows low FDG uptake, which tends to favor an inflammatory, possibly infectious, cause over neoplasm, although the morphology of the lesion has some features that are consistent with a potentially aggressive process. If enhancement of the lesion were seen, this would be consistent with a potentially aggressive lesion, prompting tissue sampling, whereas the lack of contrast enhancement, while favoring a non-malignant process, would not exclude an infection requiring treatment, again prompting a tissue diagnosis. Bronchoscopy would be a reasonable choice, given the presence of some airways associated with the lesion, but the lesion is quite peripheral and is therefore amenable to percutaneous transthoracic needle biopsy. Treating for mycobacterial infection and observing is a possibility in light of the culture results obtained from the left apical lesion, but the right lower lobe mass has a morphology that differs from the left apical mass, and the FDG uptake also differs between these two lesions, which forces the consideration that these lesions represent two coincident, but distinct, processes. Therefore, the right lower lobe mass evaluation must be approached as if this lesion were an isolated finding.

The patient underwent percutaneous transthoracic needle biopsy of the right lower lobe lesion. The material obtained at biopsy showed non-specific changes, including chronic inflammation, air space fibrin, and rare giant cells, but no evidence of malignancy, fungal organisms, or acid-fast bacilli was seen.

Given the data available, which of the following represents the most appropriate management step for the evaluation of this patient?

1. Obtain thoracoscopic surgical lung biopsy of the right lower lobe mass

2. Surgically resect the left apical lobe mass

3. Surgically resect the right lower lobe mass

4. Treat for mycobacterial and fungal infections and observe

5. Treat for mycobacterial infection and observe 


\section{Correct! \\ 5. Treat for mycobacterial infection and observe}

Treating for mycobacterial infection and observing is reasonable. Therapy for fungal infection is not indicated as all studies to date for possible fungal infection have failed to disclose such. At this point, it is reasonable to entertain the notion of obtaining a larger tissue sample in the hopes of providing a definitive diagnosis, but it remains unclear which of the two lesions, or, if one were exceedingly aggressive, both lesions, should be resected. Instead, a potential benign diagnosis that can be targeted with therapy has been suggested for the left apical lesion, so it is reasonable to institute such therapy and observe closely. The right lower lobe lesion shares some histopathological characteristics in common with the left apical lesion, although the right lower lobe lesion should be regarded as indeterminate, as no definitive histopathological diagnosis, either benign or malignant, has been established. Nevertheless, the likelihood of malignancy in this lesion has been reduced substantially in light of the lower tracer utilization at ${ }^{18} \mathrm{FDG}-\mathrm{PET}$ scan and the lack of malignancy at histopathological examination following a successful percutaneous transthoracic needle biopsy procedure, so surgical intervention, while not unreasonable, may be deferred in favor of imaging surveillance. Because the left apical lesion will be followed closely with $\mathrm{CT}$, the right lower lobe mass can be reassessed simultaneously with the same CT scan obtained to follow the left apical mass.

Some prior records were located and the patient subsequently recalled right parotic surgery for resection of a benign pleomorphic adenoma. The patient was started on clarithromycin, ethambutol, and rifampin for treatment of the MAC obtained from the left apical mass. He subsequently underwent repeat thoracic CT (images not shown) one month after the biopsy of the right lower lobe mass and institution of the antibiotic therapy, which showed no clear change in the left apical or right lower lobe lesions. Repeat thoracic CT approximately 6 months later (Figure 4) showed decrease in size of the left apical lesion but no clear change in the right lower lobe lesion.

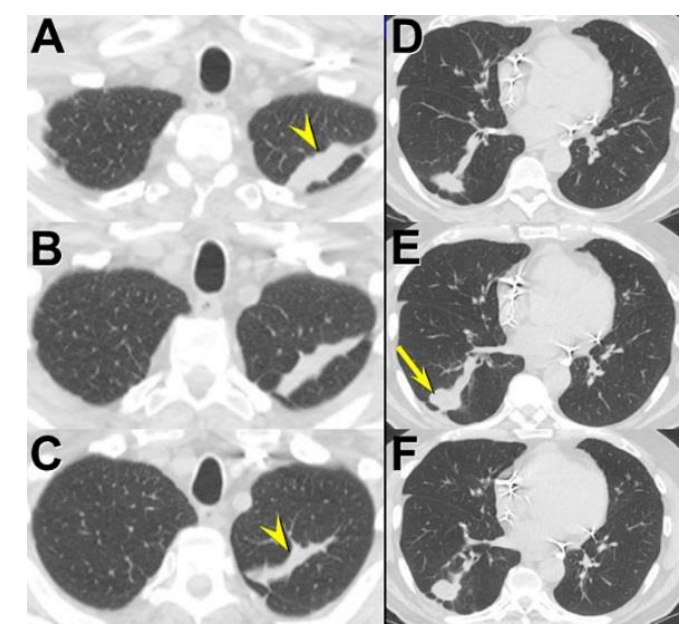

Figure 4. Axial enhanced thoracic CT displayed in lung windows 6-7 months following biopsy of the left upper lobe mass. 
Given the data available, which of the following represents the most appropriate management step for the evaluation of this patient?

1. Continue to treat for mycobacterial infection and observe

2. Obtain thoracoscopic surgical lung biopsy of the right lower lobe mass

3. Perform bronchoscopy for the right lower lobe mass

4. Perform repeat percutaneous transthoracic needle biopsy of the right lower lobe mass

5. Surgically resect the right lower lobe mass 


\section{Correct! \\ 1. Continue to treat for mycobacterial infection and observe}

While making another attempt at obtaining a tissue diagnosis is reasonable, aggressive surgical options are probably not the best choice, given that the right lower lobe mass has demonstrated some features suggesting a benign etiology (low tracer utilization at ${ }^{18}$ FDG-PET scan, the lack of malignancy at histopathological examination following a successful percutaneous transthoracic needle biopsy procedure, and, most importantly, stability at repeat imaging at just greater than 7 months). Repeat bronchoscopy would not be contraindicated, although this procedure has failed to retrieve diagnostic material for both the right lower lobe and left upper lobe mass previously. Similarly, percutaneous repeat percutaneous transthoracic needle biopsy of the right lower lobe mass failed to establish a definitive diagnosis previously and therefore repeating this procedure, given that there is no specifically suspicious area of the lesion to target that may not have been sampled previously, stands a low likelihood of providing additional useful information. It is unlikely that the parotid pleomorphic adenoma is related to the pulmonary lesions.

Repeat thoracic CT (Figure 5) performed one year after Figure 4, now exceeding 1.5 years following biopsy of the left upper lobe mass showed that the left apical lesion has resolved to mostly linear scar, and the right lower lobe lesion has decreased in size substantially, now manifesting a thin-walled cavity.

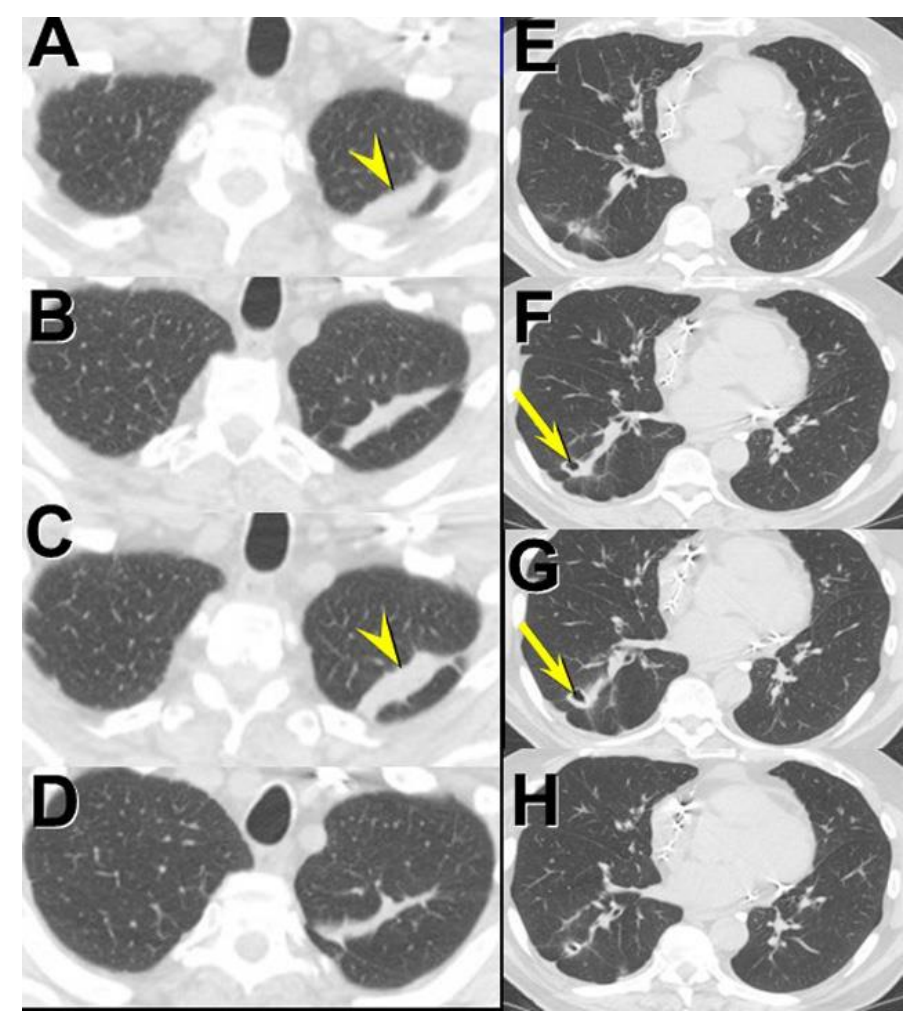

Figure 5. Axial enhanced thoracic CT displayed in lung windows, performed one year after Figure 4, now exceeding 1.5 years following biopsy of the left upper lobe mass. 
Diagnosis: Mycobacterium avium complex infection presenting as a solitary left apical pulmonary mass

\section{References}

1. Hahm CR, Park HY, Jeon K, Um SW, Suh GY, Chung MP, Kim H, Kwon OJ, Koh WJ. Solitary pulmonary nodules caused by Mycobacterium tuberculosis and Mycobacterium avium complex. Lung. 2010;188(1):25-31. [CrossRef] [PubMed]

2. Song JW, Koh WJ, Lee KS, Lee JY, Chung MJ, Kim TS, Kwon OJ. High-resolution CT findings of Mycobacterium avium-intracellulare complex pulmonary disease: correlation with pulmonary function test results. AJR Am J Roentgenol. 2008;191(4):1070. [CrossRef] [PubMed]

3. Schmeeckle KD, Yankelevitz D, Kim JW, Sartor O. Increased uptake of 18Ffluorodeoxyglucose due to Mycobacterium avium complex in a solitary pulmonary nodule. J La State Med Soc. 2008;160(3):150-2. 